Homology, Homotopy and Applications, vol.9(1), 2007, pp.451-465

\title{
SIMPLE OMEGA-CATEGORIES AND CHAIN COMPLEXES
}

\author{
RICHARD STEINER
}

(communicated by Ronald Brown)

\begin{abstract}
The category of strict omega-categories has an important full subcategory whose objects are the simple omega-categories freely generated by planar trees or by globular cardinals. We give a simple description of this subcategory in terms of chain complexes, and we give a similar description of the opposite category, the category of finite discs, in terms of cochain complexes. Berger has shown that the category of simple omegacategories has a filtration by iterated wreath products of the simplex category. We generalise his result by considering wreath products of categories of chain complexes over the simplex category.
\end{abstract}

\section{Introduction}

The category of strict $\omega$-categories has an important full subcategory $\Theta$, whose objects are the $\omega$-categories freely generated by planar trees in the sense of Batanin [1] (see also [2]). One can regard $\Theta$ as the theory of strict $\omega$-categories in the sense of universal algebra, and it has been used in the study of weak $\omega$-categories by Batanin [1] and Joyal [4]. The subcategories $\Theta_{n}$ of $\Theta$ consisting of $n$-categories for finite values of $n$ have been applied to iterated loop spaces by Berger [3].

In this paper, the objects of $\Theta$ will be called simple $\omega$-categories, following Makkai and Zawadowski [5]; they were called Batanin cells in [4], which is also the source of the notation $\Theta$. The generating structures for the objects of $\Theta$ were called globular cardinals by Street $[\mathbf{9}]$. There are several ways to describe the category $\Theta$, most of which give rather complicated descriptions of the morphisms; the main object of this paper is to give a simple description of the objects and morphisms of $\Theta$ in terms of chain complexes and chain maps. We give a new description of the generating structures for the objects of $\Theta$ in Section 2 and the description of the category $\Theta$ itself in Section 3. The method gives a similar description for the morphisms between simple $\omega$-categories and certain other $\omega$-categories, for example the oriented simplexes or orientals of Street [8] (see also [7]). The opposite category to $\Theta$ has been studied under the name of the category of finite discs [4], [5]; in Section 4

Received August 23, 2006, revised March 1, 2007; published on April 3, 2007.

2000 Mathematics Subject Classification: 18D05.

Key words and phrases: simple omega-category, augmented directed complex, category of finite discs, globular cardinal, planar tree, level-tree, simple globular set, simple omega-graph, continuously graded ordered set, Batanin cell.

Copyright (C) 2007, International Press. Permission to copy for private use granted. 
we obtain a simple description of this category in terms of cochain complexes and cochain maps. Berger [3] has shown that the subcategories $\Theta_{n}$ of $\Theta$ are equivalent to iterated wreath products of the simplex category; in Section 5 we generalise his result by studying wreath products of certain categories of chain complexes over the simplex category.

\section{Simple $\omega$-categories and graded ordered sets}

In this section we give the definition of simple $\omega$-categories, and we show that they are generated by certain graded ordered sets. The term simple $\omega$-category is due to Makkai and Zawadowski [5], but our treatment is based on the work of Street $[\mathbf{9}]$.

All of the $\omega$-categories in this paper are strict $\omega$-categories. We regard an $\omega$ category as a single set with an infinite sequence of partially defined binary composition operations, each of which makes it the set of morphisms in a small category. The composition operations are denoted $\#_{0}, \#_{1}, \ldots$, the left identity of an element $x$ under $\#_{n}$ is denoted $d_{n}^{-} x$, and the right identity of $x$ under $\#_{n}$ is denoted $d_{n}^{+} x$. The category structures commute with one another, with the special feature that

$$
d_{m}^{\beta} d_{n}^{\alpha} x=d_{n}^{\alpha} d_{m}^{\beta} x=d_{m}^{\beta} x \text { for } m<n ;
$$

in other words, if $x$ is an identity for some $\#_{m}$, then it is also an identity for $\#_{m+1}, \#_{m+2}, \ldots$ There is a final axiom saying that every element $x$ is an identity for some operation $\#_{m}$.

Simple $\omega$-categories are the free $\omega$-categories on a particular kind of globular set, called a simple globular set or a globular cardinal. We will use the following notation and definitions.

A globular set (sometimes called an $\omega$-graph) is a set in which: every element $x$ is assigned a nonnegative integer dimension $|x|$; every positive-dimensional element $|x|$ is assigned two $(|x|-1)$-dimensional elements called its source and target and here denoted $\partial^{-} x$ and $\partial^{+} x$; if $|x| \geqslant 2$ then

$$
\partial^{-} \partial^{-} x=\partial^{-} \partial^{+} x, \partial^{+} \partial^{-} x=\partial^{+} \partial^{+} x .
$$

The free $\omega$-category on a globular set is the $\omega$-category with the following presentation: the generators are the members of the globular set; if $x$ is an $n$-dimensional generator then there are relations $d_{n}^{-} x=d_{n}^{+} x=x$; if $x$ is an $n$-dimensional generator with $n>0$ then there are relations $d_{n-1}^{-} x=\partial^{-} x$ and $d_{n-1}^{+} x=\partial^{+} x$. A simple globular set or globular cardinal is a non-empty finite globular set such that the transitive closure of the relation given by $\partial^{-} x<x$ and $x<\partial^{+} x$ is a total ordering. A simple $\omega$-category is the free $\omega$-category on a simple globular set.

Since a simple globular set is finite, the transitive closure condition yields the following result.

Proposition 2.1. Let $x$ and $y$ be consecutive elements in the ordering of a simple globular set with $x<y$. Then $x=\partial^{-} y$ or $\partial^{+} x=y$. The dimensions of $x$ and $y$ differ by 1 . 
We deduce that the globular structure can be recovered from the ordering and the dimension function as follows.

Proposition 2.2. Let $x$ be a positive-dimensional element in a simple globular set. Then $\partial^{-} x$ is the last $(|x|-1)$-dimensional element before $x$ in the ordering, and $\partial^{+} x$ is the first $(|x|-1)$-dimensional element after $x$ in the ordering.

Proof. We will prove the result for $\partial^{-} x$. Let the dimension of $x$ be $n+1$. Certainly $\partial^{-} x$ is an $n$-dimensional element coming before $x$ in the ordering, so there really is a last $n$-dimensional element $a$ before $x$, and we must show that $a=\partial^{-} x$.

Let $b$ be the immediate successor of $a$. From Proposition 2.1, the dimensions of the elements $y$ such that $b \leqslant y \leqslant x$ form a consecutive set of integers. This set contains $n+1$ and does not contain $n$; therefore $|y|>n$ for $b \leqslant y \leqslant x$. In particular, Proposition 2.1 now shows that $|b|=n+1$ and $a=\partial^{-} b$.

For consecutive elements $y$ and $z$ with $b \leqslant y<z \leqslant x$ we have $|y|>n$ and $|z|>n$, and we claim further that $\left(\partial^{-}\right)^{|y|-n} y=\left(\partial^{-}\right)^{|z|-n} z$; indeed this is trivially true if $y=\partial^{-} z$, and it follows from the identity $\partial^{-} \partial^{-}=\partial^{-} \partial^{+}$if $\partial^{+} y=z$.

We now see that $y \mapsto\left(\partial^{-}\right)^{|y|-n}$ is constant on the interval $b \leqslant y \leqslant x$. In particular, $\left(\partial^{-}\right)^{|b|-n} b=\left(\partial^{-}\right)^{|x|-n} x$. Since $a=\partial^{-} b$ and $|b|=|x|=n+1$, this gives us the required equality $a=\partial^{-} x$.

It follows from Proposition 2.2 that simple globular sets are equivalent to ordered sets with suitable dimension functions; in other words they are equivalent to certain graded ordered sets. The graded ordered sets that can occur turn out to be the continuously graded ones in the sense of the following definition.

Definition 2.3. A continuously graded ordered set is a non-empty finite ordered set, together with a function assigning a nonnegative integer dimension $|x|$ to each element $x$, such that the first and last elements have dimension zero and such that consecutive elements have dimensions differing by 1 .

For example, there is a continuously graded ordered set such that the dimensions of its elements in order are

$$
0,1,2,1,2,3,4,3,2,3,4,3,2,1,0,1,0 .
$$

The main result is now as follows.

Theorem 2.4. Let $X$ be a continuously graded ordered set. Then there are welldefined functions $\partial^{-}$and $\partial^{+}$on the positive-dimensional elements of $X$ such that $\partial^{-} x$ is the last $(|x|-1)$-dimensional element preceding $x$ and $\partial^{+} x$ is the first $(|x|-1)$-dimensional element following $x$, and these functions make $X$ into a simple globular set. Every simple globular set arises from a continuously graded ordered set in this way.

Proof. By the conditions in Definition 2.3, if $x$ is a positive-dimensional element of $X$ then there is at least one $(|x|-1)$-dimensional element before $x$ and at least one $(|x|-1)$-dimensional element after $x$. Therefore the functions $\partial^{-}$and $\partial^{+}$are well-defined. If $x$ is at least 2-dimensional, then the conditions of Definition 2.3 
imply that $|y| \geqslant|x|-1$ for $\partial^{-} x \leqslant y \leqslant \partial^{+} x$, and it follows that $\partial^{\alpha} \partial^{-} x=\partial^{\alpha} \partial^{+} x$ for each sign $\alpha$; therefore $X$ is a globular set. By construction, $\partial^{-} x<x<\partial^{+} x$ for all positive-dimensional $x$; also, if $x$ and $y$ are consecutive elements with $x<y$, then $|x|=|y|-1$ or $|y|=|x|-1$, so $x=\partial^{-} y$ or $\partial^{+} x=y$. Therefore the ordering is the transitive closure obtained from the functions $\partial^{-}$and $\partial^{+}$in the way required for a simple globular set, and it follows that $X$ is indeed a simple globular set.

Conversely, let $X$ be a simple globular set. Since $\partial^{-} x<x<\partial^{+} x$ whenever $x$ has positive dimension, it follows that the first and last elements are zero-dimensional. Also, by Proposition 2.1, consecutive elements have dimensions differing by 1 , so $X$ is a continuously graded ordered set, and it follows from Proposition 2.2 that the functions $\partial^{-}$and $\partial^{+}$come from the ordering and dimensions in the way described.

This completes the proof.

Since simple globular sets are equivalent to continuously graded ordered sets, their isomorphism classes can be indexed by the non-empty finite sequences of nonnegative integers beginning and ending with 0 and with adjacent terms differing by 1 . The isomorphism classes of simple $\omega$-categories can therefore be indexed by these sequences as well. Without loss of information, a sequence of this kind can be replaced by the subsequence consisting of its maxima (terms equal to $n$ and not adjacent to $n+1$ ) and its internal minima (terms equal to $n$ adjacent to $n+1$ on both sides); for example the sequence

$$
(0,1,2,1,2,3,4,3,2,3,4,3,2,1,0,1,0)
$$

can be replaced by

$$
(2,1,4,2,4,0,1) .
$$

The original sequence can be recovered from the subsequence by interpolation. Note that the sequence $(0)$ yields the subsequence $(0)$, but the initial and final zeros are omitted from the subsequence in all other cases. The subsequences that occur are the non-empty finite sequences of nonnegative integers

$$
\left(u_{0}, v_{1}, u_{1}, v_{2}, u_{2}, \ldots, u_{k-1}, v_{k}, u_{k}\right)
$$

such that

$$
u_{0}>v_{1}, v_{1}<u_{1}, u_{1}>v_{2}, v_{2}<u_{2}, \ldots, u_{k-1}>v_{k}, v_{k}<u_{k} ;
$$

in other words they are the up-and-down vectors used to index simple $\omega$-categories by Makkai and Zawadowski ([5], 2.3). They may also be used to index planar trees with a distinguished vertex and a distinguished maximal path starting at that vertex. Indeed, given a tree with this structure, let $P_{0}, \ldots, P_{k}$ be the maximal paths starting at the distinguished vertex listed in clockwise order starting with the distinguished path, let $u_{i}$ be the number of edges in $P_{i}$, and let $v_{i}$ be the number of edges in $P_{i-1} \cap P_{i}$; then $\left(u_{0}, v_{1}, u_{1}, \ldots, u_{k}\right)$ is an up-and-down vector and every upand-down vector comes from a planar tree in this way. This explains the indexing of simple $\omega$-categories by planar trees $([\mathbf{1}],[\mathbf{2}],[\mathbf{9}])$. Berger $[\mathbf{3}]$ calls these structures level-trees. In more purely combinatorial terms, they are abstract trees with a distinguished root vertex and a total ordering at each vertex on the set of edges at that vertex pointing away from the root. To summarise, we have the following result. 
Theorem 2.5. There are explicit bijections between the following sets: the set of isomorphism classes of simple globular sets; the set of isomorphism classes of continuously graded ordered sets; the set of up-and-down vectors; the set of isomorphism classes of level-trees.

\section{Simple augmented directed complexes}

We will now recall from $[\mathbf{6}]$ the theory associating $\omega$-categories and chain complexes, and we will use it to describe the category of simple $\omega$-categories in terms of chain complexes. All our chain complexes will be augmented chain complexes of abelian groups concentrated in nonnegative dimensions. We recall that an augmented directed complex is a chain complex $K$ of this type together with a distinguished submonoid $K_{q}^{*}$ of $K_{q}$ for each chain group $K_{q}$. A morphism of augmented directed complexes from $K$ to $L$ is an augmentation-preserving chain map taking $K_{q}^{*}$ into $L_{q}^{*}$ for each $q$. The resulting category of augmented directed complexes is denoted adc.

Given an augmented directed complex $K$, we define an $\omega$-category $\nu K$ functorially as follows. The members of $\nu K$ are the double sequences

$$
\left(x_{0}^{-}, x_{0}^{+}\left|x_{1}^{-}, x_{1}^{+}\right| \cdots\right)
$$

such that

$$
\begin{aligned}
& x_{q}^{\alpha} \in K_{q}^{*}, \\
& x_{q}^{-}=x_{q}^{+}=0 \text { for all but finitely many values of } q, \\
& \epsilon x_{0}^{-}=\epsilon x_{0}^{+}=1, \\
& x_{q}^{+}-x_{q}^{-}=\partial x_{q+1}^{-}=\partial x_{q+1}^{+} .
\end{aligned}
$$

The left and right identities $d_{n}^{-} x$ and $d_{n}^{+} x$ of an element

$$
x=\left(x_{0}^{-}, x_{0}^{+}\left|x_{1}^{-}, x_{1}^{+}\right| \cdots\right)
$$

are given by

$$
d_{n}^{\alpha} x=\left(x_{0}^{-}, x_{0}^{+}|\cdots| x_{n-1}^{-}, x_{n-1}^{+}\left|x_{n}^{\alpha}, x_{n}^{\alpha}\right| 0,0 \mid \cdots\right) .
$$

If $d_{n}^{+} x=d_{n}^{-} y$, say $d_{n}^{+} x=d_{n}^{-} y=z$, then the composite $x \#_{n} y$ is $x-z+y$.

We are especially interested in augmented directed complexes with bases. These are augmented directed complexes of free abelian groups with prescribed bases such that the distinguished submonoids are generated, as monoids, by the prescribed basis elements. It is convenient to work with the union of the bases for the chain groups, a single graded set which we regard as the basis for the entire augmented directed complex. In effect, we are identifying a chain complex $K$ with the direct sum

$$
K_{0} \oplus K_{1} \oplus K_{2} \oplus \cdots .
$$

We note that an augmented directed complex can have at most one basis, because a free abelian monoid has a unique basis (consisting of its indecomposable non-zero elements). A globular set $X$ generates an augmented directed complex with basis 
equal to the graded set $X$ as follows: if $x \in X$ and $|x|=0$, then $\epsilon x=1$; if $x \in X$ and $|x|>0$, then $\partial x=\partial^{+} x-\partial^{-} x$. We are interested in the cases when $X$ is a simple globular set.

Definition 3.1. A simple augmented directed complex is an augmented directed complex generated by a simple globular set.

If $x$ is a positive-dimensional basis element in a simple augmented directed complex, then $\partial^{-} x$ and $\partial^{+} x$ are distinct basis elements. More generally, let $K$ be any augmented directed complex with a basis and let $x$ be any chain in $K$; then we will write

$$
\partial x=\partial^{+} x-\partial^{-} x,
$$

such that $\partial^{-} x$ and $\partial^{+} x$ are sums of basis elements with no common terms. This means that $\partial^{+} x$ and $\partial^{-} x$ are the 'positive and negative parts of $\partial x$ '. The basis for $K$ is called unital if

$$
\epsilon\left(\partial^{-}\right)^{|b|} b=\epsilon\left(\partial^{+}\right)^{|b|} b=1
$$

for every basis element $b$. If the basis is unital, then we associate an element $\langle b\rangle$ of $\nu K$ called an atom to every basis element $b$ as follows:

$$
\langle b\rangle=\left(\left(\partial^{-}\right)^{|b|} b,\left(\partial^{+}\right)^{|b|} b|\cdots| \partial^{-} b, \partial^{+} b|b, b| 0,0 \mid \cdots\right) .
$$

In particular, the basis of a simple augmented directed complex is unital, because $\left(\partial^{\alpha}\right)^{|b|} b$ is a basis element for every basis element $b$ and because 0 -dimensional basis elements have augmentation 1 . If $b$ is a positive-dimensional basis element in a simple augmented directed complex, then $\partial^{-} b$ and $\partial^{+} b$ are basis elements, so there are atoms $\left\langle\partial^{-} b\right\rangle$ and $\left\langle\partial^{+} b\right\rangle$. The identities $\partial^{\alpha} \partial^{-}=\partial^{\alpha} \partial^{+}$then give the following result.

Proposition 3.2. If $b$ is an $n$-dimensional basis element in a simple augmented directed complex with $n>0$, then

$$
d_{n-1}^{-}\langle b\rangle=\left\langle\partial^{-} b\right\rangle, d_{n-1}^{+}\langle b\rangle=\left\langle\partial^{+} b\right\rangle .
$$

The main results of [6] apply to augmented directed complexes with bases when the bases are unital and are also loop-free, in the following sense: for $q \geqslant 0$ there is a partial ordering $<_{q}$ on the basis elements of degree at least $q$ such that $a<_{q} b$ if $a$ is a term in $\left(\partial^{-}\right)^{|b|-q} b$ with $|b|>q$ or if $b$ is a term in $\left(\partial^{+}\right)^{|a|-q} a$ with $|a|>q$. In practice we usually find that the basis is strongly loop-free; in this case there is a partial ordering $<_{\mathbf{N}}$ on the entire basis such that $a<_{\mathbf{N}} b$ if $a$ is a term in $\partial^{-} b$ or if $b$ is a term in $\partial^{+} a$. A strongly loop-free basis is loop-free, as the terminology suggests, because one can get suitable partial orderings $<_{q}$ by restricting the partial ordering $<_{\mathbf{N}}$. In particular, the basis of a simple augmented directed complex is strongly loop-free, because the total ordering of the basis given by its structure as a continuously graded ordered set has the property required for $<_{\mathbf{N}}$.

Let $K$ be an augmented directed complex with a loop-free unital basis. The first main result of [6] (Theorem 6.1) says that the $\omega$-category $\nu K$ has a presentation of the following kind: the generators are the atoms; for each atom $\langle b\rangle$ such that $|b|=n$, 
there are relations $d_{n}^{-}\langle b\rangle=d_{n}^{+}\langle b\rangle=\langle b\rangle$; for each atom $\langle b\rangle$ such that $|b|=n>0$, there are relations expressing $d_{n-1}^{-}\langle b\rangle$ and $d_{n-1}^{+}\langle b\rangle$ as iterated composites of atoms (if $d_{n-1}^{\alpha}\langle b\rangle$ can be expressed as an iterated composite of atoms in more than one way, then we can choose any such expression). When $K$ is a simple augmented directed complex, we can use the formulae $d_{n-1}^{\alpha}\langle b\rangle=\left\langle\partial^{\alpha} b\right\rangle$ of Proposition 3.2 and we get the following result.

Proposition 3.3. If $K$ is a simple augmented directed complex, then $\nu K$ is the simple $\omega$-category generated by the basis for $K$.

The second main result of [6] (Theorem 5.11) says that the functor $\nu$ restricted to augmented directed complexes with loop-free unital bases is a fully faithful embedding in the category of $\omega$-categories. Since simple augmented directed complexes have loop-free unital bases, a further restriction produces the following result.

Theorem 3.4. The restriction of $\nu$ to the category of simple augmented directed complexes is a fully faithful embedding with image equivalent to the category $\Theta$ of simple $\omega$-categories.

From this theorem we get a simple description of a category equivalent to $\Theta$. The objects are augmented chain complexes of free abelian groups with prescribed non-empty finite ordered bases $b_{0}, \ldots, b_{p}$ such that $\left|b_{0}\right|=\left|b_{p}\right|=0$ and such that $\left|b_{q}\right|-\left|b_{q-1}\right|= \pm 1$ for $1 \leqslant q \leqslant p$. The augmentation is such that $\epsilon b_{q}=1$ for $\left|b_{q}\right|=0$. The boundary is such that $\partial b_{q}=\partial^{+} b_{q}-\partial^{-} b_{q}$ for $\left|b_{q}\right|>0$, where $\partial^{+} b_{q}$ is the last $\left(\left|b_{q}\right|-1\right)$-dimensional basis element before $b_{q}$ and where $\partial^{-} b_{q}$ is the first $\left(\left|b_{q}\right|-1\right)$ dimensional basis element after $b_{q}$. The morphisms are the augmentation-preserving chain maps taking sums of prescribed basis elements to sums of prescribed basis elements.

We will now show how to get a more combinatorial description of these morphisms. Let $K$ be a simple augmented directed complex. A finite, possibly empty, sequence of basis elements $\left(a_{1}, \ldots, a_{p}\right)$ will be called ordered if $a_{1} \leqslant a_{2} \leqslant \cdots \leqslant a_{p}$. To an ordered sequence of $n$-dimensional basis elements $\left(a_{1}, \ldots, a_{p}\right)$ we will associate the sum $a_{1}+\cdots+a_{p}$; in this way the elements of $K_{n}^{*}$ are identified with the ordered sequences of $n$-dimensional basis elements.

A sequence of $n$-dimensional basis elements $\left(a_{1}, \ldots, a_{p}\right)$ will be called separated if it is ordered and if each consecutive pair of terms encloses a basis element of lower dimension; in particular a sequence of 0-dimensional basis elements is separated if and only if it is empty or a singleton. Note that the members of a separated sequence are distinct.

The set of separated sequences of $n$-dimensional elements will be partially ordered such that $\left(a_{1}^{\prime}, \ldots, a_{p}^{\prime}\right) \leqslant\left(a_{1}^{\prime \prime}, \ldots, a_{q}^{\prime \prime}\right)$ if and only if the following conditions hold:

$$
\begin{aligned}
& p=q ; \\
& a_{i}^{\prime} \leqslant a_{i}^{\prime \prime} \text { for } 1 \leqslant i \leqslant p ;
\end{aligned}
$$

for $1 \leqslant i \leqslant p$ the elements between $a_{i}^{\prime}$ and $a_{i}^{\prime \prime}$ are at least $n$-dimensional.

Equivalently, for a separated sequence of $n$-dimensional elements $a^{\prime}$ and $a^{\prime \prime}$, one has $a^{\prime} \leqslant a^{\prime \prime}$ if and only if $a^{\prime \prime}-a^{\prime}$ is the boundary of a member of $K_{n+1}^{*}$. 
Let

$$
a^{\prime}=\left(a_{1}^{\prime}, \ldots, a_{p}^{\prime}\right), \quad a^{\prime \prime}=\left(a_{1}^{\prime \prime}, \ldots, a_{p}^{\prime \prime}\right)
$$

be separated sequences of $n$-dimensional elements such that $a^{\prime} \leqslant a^{\prime \prime}$. For $1 \leqslant i \leqslant p$, let $\left\{a_{i, 0}, \ldots, a_{i, q(i)}\right\}$ be the set of $n$-dimensional elements $a$ with $a_{i}^{\prime} \leqslant a \leqslant a_{i}^{\prime \prime}$ indexed so that

$$
a_{i}^{\prime}=a_{i, 0}<a_{i, 1}<\cdots<a_{i, q(i)}=a_{i}^{\prime \prime} .
$$

A sequence of $(n+1)$-dimensional elements will be said to bridge $a^{\prime}$ and $a^{\prime \prime}$ if it has the form

$$
\left(b_{1,1}, \ldots, b_{1, q(1)}, \ldots, b_{p, 1}, \ldots, b_{p, q(p)}\right)
$$

such that $a_{i, j-1}<b_{i, j}<a_{i, j}$ for all $i$ and $j$. Equivalently, $b$ bridges $a^{\prime}$ and $a^{\prime \prime}$ if and only if $b$ is a member of $K_{n+1}^{*}$ such that $\partial b=a^{\prime \prime}-a^{\prime}$. Note that a sequence bridging two separated sequences is itself separated.

In these terms we get the following results.

Theorem 3.5. Let $f: K \rightarrow L$ be a morphism of augmented directed complexes between simple augmented directed complexes $K$ and $L$, and let a be a basis element in $K$. Then $f a$ is a separated sequence of basis elements in $L$.

Proof. The proof is by induction on $|a|$.

If $|a|=0$, then $\epsilon f a=\epsilon a=1$, so $f a$ is a singleton and is therefore separated.

If $|a|>0$, then $f \partial^{-} a$ and $f \partial^{+} a$ are separated by the inductive hypothesis and $f a$ bridges $f \partial^{-} a$ and $f \partial^{+} a$ because $\partial f a=f \partial a=f \partial^{+} a-f \partial^{-} a$, so $f a$ is separated.

This completes the proof.

Theorem 3.6. Let $K$ and $L$ be simple augmented directed complexes and let $f$ be a function assigning a separated sequence of $n$-dimensional basis elements in $L$ to every $n$-dimensional basis element in $K$. Then $f$ extends to a morphism of augmented directed complexes if and only if the following conditions are satisfied.

(i) Let $a_{1}, \ldots, a_{p}$ be the zero-dimensional basis elements in $K$ indexed so that $a_{1}<\cdots<a_{p}$. Then the $f a_{i}$ are singleton sequences such that $f a_{1} \leqslant \cdots \leqslant f a_{p}$.

(ii) For $n>0$, let $a^{\prime}$ and $a^{\prime \prime}$ be $(n-1)$-dimensional basis elements in $K$ such that $a^{\prime}<a^{\prime \prime}$ and such that the elements between $a^{\prime}$ and $a^{\prime \prime}$ have dimension at least $n$. Let $a_{1}, \ldots, a_{p}$ be the $n$-dimensional elements between $a^{\prime}$ and $a^{\prime \prime}$ indexed so that $a^{\prime}<a_{1}<\cdots<a_{p}<a^{\prime \prime}$. Then the $f a_{i}$ are sequences bridging $f a^{\prime}$ and $f a^{\prime \prime}$ such that $f a_{1} \leqslant \cdots \leqslant f a_{p}$.

Proof. Condition (i) is equivalent to saying that $f$ is augmentation-preserving and that $f \partial K_{1}^{*} \subset \partial L_{1}^{*}$. Condition (ii) is equivalent to saying that $\partial f=f \partial$ on $K_{n}$ and that $f \partial K_{n+1}^{*} \subset \partial L_{n+1}^{*}$. These conditions are clearly necessary and sufficient for $f$ to give a morphism of simple augmented directed complexes.

From Theorem 3.5, because the members of separated sequences are distinct, morphisms of augmented directed complexes between simple augmented directed complexes can be regarded as functions taking basis elements to sets of basis elements. Theorem 3.6 therefore gives a combinatorial description of the morphisms 
equivalent to the original definition [4] (see also [5]). Theorem 3.6 also shows that the morphisms can be constructed inductively: if one has constructed a morphism in dimensions less than $n$ satisfying the given conditions, then one can always extend it to higher dimensions.

\section{The category of finite discs}

Makkai and Zawadowski [5] have shown that Joyal's category of finite discs [4] is the opposite category to the category of simple $\omega$-categories. Since morphisms between simple $\omega$-categories can be represented by chain maps between finitely generated free chain complexes, it follows that morphisms between finite discs can be represented by cochain maps between the dual cochain complexes. We will now give the details.

Recall that simple augmented directed complexes are augmented chain complexes of finitely generated free abelian groups with prescribed bases. Using dual bases, we see that the dual cochain complexes are coaugmented cochain complexes of finitely generated free abelian groups also with prescribed bases. It is clear that the duals of the augmentation-preserving chain maps are the coaugmentation-preserving cochain maps. Also, a chain map takes sums of prescribed basis elements to sums of prescribed basis elements if and only if its dual does the same for the dual bases (in matrix terms, the entries of a matrix are nonnegative if and only if the entries of its transpose are nonnegative). We therefore get an easily described category equivalent to the category of finite discs as follows.

The objects are coaugmented cochain complexes of free abelian groups with prescribed non-empty finite bases $c_{0}, \ldots, c_{p}$ such that $\left|c_{0}\right|=\left|c_{p}\right|=0$ and such that $\left|c_{q}\right|-\left|c_{q-1}\right|= \pm 1$ for $1 \leqslant q \leqslant p$. The coaugmentation $\eta$ is such that $\eta(1)$ is the sum of the 0 -dimensional basis elements. The coboundary $\delta$ is given by

$$
\delta c_{q}=\delta^{+} c_{q}-\delta^{-} c_{q}
$$

where $\delta^{+} c_{q}$ is the sum of the $\left(\left|c_{q}\right|+1\right)$-dimensional elements $c_{r}$ with $r<q$ such that $\left|c_{t}\right|>\left|c_{q}\right|$ for $r \leqslant t<q$, and where $\delta^{-} c_{q}$ is the sum of the $\left(\left|c_{q}\right|+1\right)$-dimensional elements $c_{s}$ with $q<s$ such that $\left|c_{t}\right|>\left|c_{q}\right|$ for $q<t \leqslant s$. The morphisms are the coaugmentation-preserving cochain maps taking sums of prescribed basis elements to sums of prescribed basis elements.

\section{Wreath products over the simplex category}

For $n=0,1,2, \ldots$, let $\Theta_{n}$ be the full subcategory of $\Theta$ consisting of the $n$ categories. We recall that an $n$-category is an $\omega$-category in which every element is an identity for $\#_{n}$; a simple $\omega$-category is therefore an object of $\Theta_{n}$ for which the

dimensions in the associated continuously graded ordered set do not exceed $n$. The categories $\Theta_{n}$ form a chain

$$
\Theta_{0} \subset \Theta_{1} \subset \Theta_{2} \subset \cdots
$$


and their union is the entire category $\Theta$. Berger $[\mathbf{3}]$ has shown how to construct $\Theta_{n}$ by using the functor

$$
\mathcal{A} \mapsto \Delta 乙 \mathcal{A}
$$

from categories to categories which takes each category to its wreath product over the simplex category. He does this by constructing equivalences $\Delta \curlyvee \Theta_{n-1} \rightarrow \Theta_{n}$, from which it follows that $\Theta_{n}$ is equivalent to the $n$-fold iterated wreath product

$$
\Delta \imath \cdots \imath \Delta \text {. }
$$

We will generalise his result by constructing a functor

$$
V: \Delta \text { idc } \rightarrow \text { adc }
$$

and showing that its restriction to an appropriate subcategory is fully faithful.

We begin by recalling the definition of the wreath product category $\Delta$ \& for an arbitrary category $\mathcal{A}$. The objects of $\Delta \imath \mathcal{A}$ are the pairs $(m, A)$, where $m$ is a nonnegative integer and where $A=\left(A^{1}, \ldots, A^{m}\right)$ is an ordered $m$-tuple of objects of $\mathcal{A}$. The morphisms in $\Delta \prec \mathcal{A}$ from $(m, A)$ to $(n, B)$ are the pairs $(\phi, f)$, where $\phi=(\phi(0), \ldots, \phi(m))$ is an ordered $(m+1)$-tuple of integers with

$$
0 \leqslant \phi(0) \leqslant \phi(1) \leqslant \cdots \leqslant \phi(m) \leqslant n
$$

and where

$$
f=\left(f_{1}^{\phi(0)+1}, \ldots, f_{1}^{\phi(1)}, f_{2}^{\phi(1)+1}, \ldots, f_{2}^{\phi(2)}, \ldots, f_{m}^{\phi(m-1)+1}, \ldots, f_{m}^{\phi(m)}\right)
$$

is an ordered $[\phi(m)-\phi(0)]$-tuple of morphisms $f_{i}^{j}: A^{i} \rightarrow B^{j}$ in $\mathcal{A}$. Composition in $\Delta \prec \mathcal{A}$ is given by

$$
(\psi, g) \circ(\phi, f)=(\psi \circ \phi, g \circ f),
$$

where $\psi \circ \phi(i)=\psi(\phi(i))$ and where $(g \circ f)_{i}^{k}=g_{j}^{k} \circ f_{i}^{j}$ for

$$
\psi \circ \phi(i-1) \leqslant \psi(j-1) \leqslant k-1<k \leqslant \psi(j) \leqslant \psi \circ \phi(i) .
$$

If $\mathcal{A}$ is the category with one object and one morphism, then one sees that $\Delta l \mathcal{A}$ is the simplex category $\Delta$ itself. It is clear that the wreath product construction $\Delta$ ? is functorial.

We will now construct the functor

$$
V: \Delta \zeta \text { adc } \rightarrow \text { adc. }
$$

As before, it is convenient to regard an augmented directed complex $K$ as the direct sum of its chain groups $K_{q}$, so that

$$
K=K_{0} \oplus K_{1} \oplus \cdots \text {. }
$$

Let $(m, K)$ be an object of $\Delta$ r adc with $K=\left(K^{1}, \ldots, K^{m}\right)$. For $0 \leqslant i \leqslant m$, let $\mathbf{Z} p^{i}$ be a free abelian group with a single basis element $p^{i}$. For $1 \leqslant i \leqslant m$, let $s K^{i}$ be an abelian group isomorphic to $K^{i}$ and let $s: K^{i} \rightarrow s K^{i}$ be an isomorphism. Then

$$
V(m, K)=\mathbf{Z} p^{0} \oplus s K^{1} \oplus \mathbf{Z} p^{1} \oplus \cdots \oplus \mathbf{Z} p^{m-1} \oplus s K^{m} \oplus \mathbf{Z} p^{m}
$$

with the following structure. The degrees are given by $\left|p^{i}\right|=0$ and $|s x|=|x|+1$. The boundary is given as follows: if $x \in K^{i}$ with $|x|>0$ then $\partial s x=s \partial x$; if $x \in K^{i}$ 
with $|x|=0$, then

$$
\partial s x=(\epsilon x)\left(p^{i}-p^{i-1}\right)
$$

and finally $\partial p^{i}=0$. The augmentation is given by $\epsilon p^{i}=1$. The distinguished submonoid of $V(m, K)$ is generated by the elements $p^{i}$ and by the images under $s$ of the distinguished submonoids of the $K^{i}$.

Now let $(\phi, f):(m, K) \rightarrow(n, L)$ be a morphism in $\Delta$ ? adc. Then

$$
V(\phi, f): V(m, K) \rightarrow V(n, L)
$$

is the homomorphism given as follows: if $0 \leqslant i \leqslant m$, then $p^{i} \mapsto p^{\phi(i)}$; if $x \in K^{i}$, then

$$
s x \mapsto \sum_{\phi(i-1)<j \leqslant \phi(i)} s f_{i}^{j} x .
$$

It is straightforward to check that $V(\phi, f)$ is a morphism of augmented directed complexes, and that $V$ is a functor.

Example 5.1. Let $K^{1}, \ldots, K^{m}$ be simple augmented directed complexes and let the sequences of dimensions for the underlying continuously graded ordered sets be $t^{1}, \ldots, t^{m}$. Then $V(m, K)$ is a simple augmented directed complex such that the sequence of dimensions for the underlying continuously graded ordered set is

$$
\left(0, s t^{1}, 0, s t^{2}, 0, \ldots, 0, s t^{k}, 0\right),
$$

where the sequence $s t^{i}$ is obtained from the sequence $t^{i}$ by adding 1 to each term.

Example 5.2. Let $K^{1}, \ldots, K^{m}$ be the cellular chain complexes of cell complexes $X^{i}$ with prescribed orientations for the cells, made into augmented directed complexes by taking the oriented cells as bases. Let $I$ be the closed interval $[0,1]$, let $P^{0}, \ldots, P^{m}$ be one-point spaces, and let $V(m, X)$ be obtained from the disjoint union of the spaces

$$
P^{0}, X^{1} \times I, P^{1}, \ldots, P^{m-1}, X^{m} \times I, P^{m}
$$

by identifying $X^{i} \times\{0\}$ with $P^{i-1}$ and $X^{i} \times\{1\}$ with $P^{i}$. Thus $V(m, X)$ is a chain obtained by joining the unreduced suspensions of the spaces $X^{i}$ together; alternatively, $V(m, X)$ is the homotopy colimit of the unique diagram of the form

$$
P^{0} \leftarrow X^{1} \rightarrow P^{1} \leftarrow \cdots \rightarrow P^{m-1} \leftarrow X^{m} \rightarrow P^{m}
$$

There is an obvious cell structure on $V(m, X)$ : the cells are the products $c \times I$ for $c$ a cell in some $X^{i}$ together with the points $P^{i}$. If one orientates the cells correctly, then $V(m, K)$ becomes the cellular chain complex of $V(m, X)$.

The functor $V$ behaves well on bases.

Proposition 5.3. Let $(m, K)$ be an object of $\Delta$ idc such that the augmented directed complexes $K^{i}$ have bases. Then $V(m, K)$ has a basis. If the bases for the $K^{i}$ are unital, then the basis for $V(m, K)$ is unital. If the bases for the $K^{i}$ are loop-free, then the basis for $V(m, K)$ is loop-free. If the bases for the $K^{i}$ are strongly loop-free, then the basis for $V(m, K)$ is strongly loop-free. 
Proof. It is straightforward to check that $V(m, K)$ has a basis consisting of the zero-dimensional elements $p^{i}$ together with the images under $s$ of the bases for the terms $K^{i}$.

Suppose that the bases for the $K^{i}$ are unital. By construction, $\epsilon p^{i}=1$. If $b$ is a basis element for $K^{i}$, then

$$
\epsilon\left(\partial^{-}\right)^{|s b|} s b=\epsilon \partial^{-}\left(\partial^{-}\right)^{|b|} s b=\epsilon \partial s\left(\partial^{-}\right)^{|b|} b=\epsilon\left[\epsilon\left(\partial^{-}\right)^{|b|} b\right] p^{i-1}=\epsilon\left(1 p^{i-1}\right)=1
$$

and $\epsilon\left(\partial^{+}\right)^{|s b|} s b=\epsilon p^{i}=1$ similarly. Therefore the basis for $V(m, K)$ is unital.

Suppose that the bases for the $K^{i}$ are loop-free, with partial orderings $<_{q}$. For $q>0$, one gets a partial ordering $<_{q}$ in $V(m, K)$ as required for loop-freeness by taking the union of the images of the partial orderings $<_{q-1}$ under the isomorphisms $s: K^{i} \rightarrow s K^{i}$. One also gets a partial ordering $<_{0}$ in $V(m, K)$ with the required property as follows:

$$
\begin{aligned}
& p^{i}<_{0} p^{j} \text { for } i<j, \\
& p^{i}<_{0} s b \text { for } b \in K^{j} \text { with } i<j, \\
& s a<_{0} p^{j} \text { for } a \in K^{i} \text { with } i \leqslant j, \\
& s a<_{0} s b \text { for } a \in K^{i} \text { and } b \in K^{j} \text { with } i<j .
\end{aligned}
$$

Therefore the basis for $V(m, K)$ is loop-free.

Now suppose that the bases for the $K^{i}$ are strongly loop-free with partial orderings $<_{\mathbf{N}}$. Then the basis for $V(m, K)$ is strongly loop-free under the partial ordering $<_{\mathbf{N}}$ given by

$$
\begin{aligned}
& p^{i}<_{\mathbf{N}} p^{j} \text { for } i<j, \\
& p^{i}<_{\mathbf{N}} s b \text { for } b \in K^{j} \text { with } i<j, \\
& s a<_{\mathbf{N}} p^{j} \text { for } a \in K^{i} \text { with } i \leqslant j, \\
& s a<_{\mathbf{N}} s b \text { for } a \in K^{i} \text { and } b \in K^{j} \text { with } i<j, \\
& s a<\mathbf{N} s b \text { for } a<_{\mathbf{N}} b \text { in some } K^{i} .
\end{aligned}
$$

This completes the proof.

Let $\Phi$ be the full subcategory of adc consisting of non-zero augmented directed chain complexes with loop-free unital bases. Because of Proposition 5.3, the functor $V$ maps $\Delta \succ \Phi$ into $\Phi$, and the main result on the functor $V$ says that the restriction

$$
V: \Delta \imath \Phi \rightarrow \Phi
$$

is fully faithful. We need two subsidiary results.

Proposition 5.4. Let $K$ be a non-zero augmented directed complex with a unital basis. Then $K$ has at least one zero-dimensional basis element, and every zerodimensional basis element has augmentation 1.

Proof. Since $K$ is non-zero, there is at least one basis element $b$. For this element we have $\epsilon\left(\partial^{-}\right)^{|b|} b=1$, so $\left(\partial^{-}\right)^{|b|} b$ is a non-zero chain of dimension zero. It follows that there is at least one zero-dimensional basis element. Finally, if $a$ is a zerodimensional basis element then $\epsilon a=\epsilon\left(\partial^{-}\right)^{|a|} a=1$. 
Proposition 5.5. Let $K$ and $L$ be augmented directed complexes with bases such that the basis for $L$ is loop-free and unital. If $f: K \rightarrow L$ is a chain map taking sums of basis elements to sums of basis elements such that $\epsilon \circ f=0$, then $f=0$.

Proof. It suffices to show that $f a=0$ for every basis element $a$ in $K$, and we will use induction on $|a|$.

Suppose that $|a|=0$. Then

$$
f a=b_{1}+\cdots+b_{k}
$$

for some basis elements $b_{i}$ in $K$. We have $\epsilon b_{i}=1$ for each $i$, because the basis for $L$ is unital, so $\epsilon f a=k$. But $\epsilon f a=0$, so $k=0$, which means that $f a=0$.

Now suppose that $|a|=q+1>0$. Again we have

$$
f a=b_{1}+\cdots+b_{k}
$$

for some basis elements $b_{i}$, and we must show that $k=0$. Suppose therefore that $k>0$. Since the basis for $L$ is loop-free, we can choose $b_{i}$ such that $b_{j}<_{q} b_{i}$ is not true for any $j$. Since $\epsilon\left(\partial^{-}\right)^{q+1} b_{i}=1$, we must have $\partial^{-} b_{i} \neq 0$. But a basis element which is a term in $\partial^{-} b_{i}$ cannot be cancelled by a term in $\partial^{+} b_{j}$ for any $j$, because we do not have $b_{j}<_{q} b_{i}$, so we get

$$
f \partial a=\partial f a=\partial b_{1}+\cdots+\partial b_{k} \neq 0,
$$

contrary to the inductive hypothesis. Therefore $f a=0$.

This completes the proof.

The main result is now as follows.

Theorem 5.6. Let $\Phi$ be the full subcategory of adc consisting of the non-zero augmented directed complexes with loop-free unital bases. Then the functor

$$
V: \Delta \curlywedge \Phi \rightarrow \Phi
$$

is fully faithful.

Proof. Let $(m, K)$ and $(n, L)$ be objects of $\Delta \curlywedge \Phi$, and let

$$
F: V(m, K) \rightarrow V(n, L)
$$

be a morphism in $\Phi$. We must show that $F=V(\phi, f)$ for a unique morphism $(\phi, f):(m, K) \rightarrow(n, L)$ in $\Delta \imath \Phi$.

As an abelian group, $V(m, K)$ is generated by the elements $p^{i}$ and the subgroups $s K^{i}$, so $F$ is determined by its values on $p^{i}$ and on $s K^{i}$. Since $F$ is an augmentationpreserving chain map taking sums of basis elements to sums of basis elements, $F p^{i}$ must be a sum of zero-dimensional basis elements such that $\epsilon F p^{i}=1$. This forces $F p^{i}$ to be a single basis element, so

$$
F p^{i}=p^{\phi(i)}
$$

for some $\phi(i)$ with $0 \leqslant \phi(i) \leqslant n$. Also, since $F$ is a chain map taking sums of basis 
elements to sums of basis elements, we must have

$$
F s x=\sum_{j=1}^{n} s f_{i}^{j} x \text { for } x \in K^{i}
$$

where the $f_{i}^{j}: K^{i} \rightarrow L^{j}$ are uniquely determined chain maps taking sums of basis elements to sums of basis elements. To show that $F=V(\phi, f)$ for a unique morphism $(\phi, f):(m, K) \rightarrow(n, L)$ in $\Delta \curlywedge \Phi$, it now suffices to show that

$$
\phi(0) \leqslant \phi(1) \leqslant \cdots \leqslant \phi(m),
$$

that $f_{i}^{j}$ is augmentation-preserving for $\phi(i-1)<j \leqslant \phi(i)$, and that $f_{i}^{j}=0$ otherwise.

We first show that $\phi(i-1) \leqslant \phi(i)$, using the assumption that $K^{i} \neq 0$. By Proposition 5.4 , there is a zero-dimensional basis element $a$ in $K^{i}$, and $\epsilon a=1$. It follows that

$$
F \partial s a=F(\epsilon a)\left(p^{i}-p^{i-1}\right)=p^{\phi(i)}-p^{\phi(i-1)}
$$

and that

$$
\partial F s a=\partial \sum_{j=1}^{n} s f_{i}^{j} a=\sum_{j=1}^{n}\left(\epsilon f_{i}^{j} a\right)\left(p^{j}-p^{j-1}\right) .
$$

Since $f_{i}^{j} a$ is a sum of basis elements, we must have $\epsilon f_{i}^{j} a \geqslant 0$ for all $j$. Since $F \partial s a=$ $\partial F s a$, we must have $\phi(i-1) \leqslant \phi(i)$.

Next we compute $\epsilon \circ f_{i}^{j}$. Let $x$ be a zero-dimensional chain in $K^{i}$. Equating $F \partial s x$ and $\partial F s x$ just as in the previous paragraph, we get

$$
(\epsilon x)\left(p^{\phi(i)}-p^{\phi(i-1)}\right)=\sum_{j=1}^{n}\left(\epsilon f_{i}^{j} x\right)\left(p^{j}-p^{j-1}\right) .
$$

For $\phi(i-1)<j \leqslant \phi(i)$ we get $\epsilon f_{i}^{j} x=\epsilon x$, so that $f_{i}^{j}$ is augmentation-preserving, and for other values of $j$ we get $\epsilon f_{i}^{j} x=0$, so that $f_{i}^{j}=0$ by Proposition 5.5.

This completes the proof.

Let us now consider the categories $\Theta_{n}$ in the filtration of $\Theta$ given by

$$
\Theta_{0} \subset \Theta_{1} \subset \Theta_{2} \subset \cdots
$$

The category $\Theta$ is equivalent to a full subcategory of adc, and its objects are non-zero augmented directed complexes with loop-free unital bases. It follows from Theorem 5.6 that $V$ induces a fully faithful functor from $\Delta \imath \Theta$ to strict $\omega$-categories. From Example 5.1 we see that this functor restricts to an equivalence $\Delta \imath \Theta_{n-1} \cong$ $\Theta_{n}$; this equivalence is also visible in Theorem 3.6. Now an object of $\Theta_{0}$ is an augmented directed complex with a basis consisting of a single zero-dimensional element of augmentation 1. Clearly if $K$ and $L$ are objects of $\Theta_{0}$, then there is a unique morphism of augmented directed complexes from $K$ to $L$. It follows that $\Theta_{0}$ is equivalent to the category with a single object and a single morphism. For $n>0$ it then follows that $\Theta_{n}$ is equivalent to the $n$-fold iterated wreath product $\left.\Delta\right\} \cdots \imath \Delta$; we have therefore recovered Berger's result ([3], Theorem 3.7). The inclusion functor 
$\Theta_{0} \rightarrow \Theta_{1}$ is equivalent to the functor $\Theta_{0} \rightarrow \Delta \curlywedge \Theta_{0}$ sending the objects of $\Theta_{0}$ to the unique object of the form $(0, A)$ in $\Delta \prec \Theta_{0}$, and the inclusions $\Theta_{n-1} \rightarrow \Theta_{n}$ are obtained from this by repeated application of the wreath product functor. Up to equivalence, the categories $\Theta_{n}$ and the inclusion functors $\Theta_{n-1} \rightarrow \Theta_{n}$ can therefore be expressed entirely in terms of the simplex category and wreath products. The entire category $\Theta$ can then be expressed in this way as well, since it is the colimit of the sequence

$$
\Theta_{0} \rightarrow \Theta_{1} \rightarrow \Theta_{2} \rightarrow \cdots
$$

Remark 5.7. In particular, we have an isomorphism $\Delta \cong \Theta_{1}$ giving a fully faithful embedding of the simplex category $\Delta$ in the category of augmented directed complexes adc. There is a completely different embedding of $\Delta$ in adc sending the objects of $\Delta$ to the simplicial chain complexes of the standard simplexes (see [7]). This embedding is not full. The corresponding $\omega$-categories are Street's orientals (see $[\mathbf{8}])$. The oriental corresponding to the $n$-dimensional simplex is a simple $\omega$ category only for $n=0$ and for $n=1$.

\section{References}

[1] M.A. Batanin, Monoidal globular categories as a natural environment for the theory of weak n-categories, Adv. Math. 136 (1998), no. 1, 39-103.

[2] M.A. Batanin and R. Street, The universal property of the multitude of trees. Category theory and its applications, J. Pure Appl. Algebra 154 (2000), no. 1-3, 3-13.

[3] C. Berger, Iterated wreath product of the simplex category and iterated loop spaces, Adv. Math., to appear.

[4] A. Joyal, Disks, duality and $\theta$-categories, unpublished, 1997.

[5] M. Makkai and M. Zawadowski, Duality for simple $\omega$-categories and disks, Theory Appl. Categ. 8 (2001), 114-243.

[6] R. Steiner, Omega-categories and chain complexes, Homology, Homotopy Appl. 6 (2004), no. 1, 175-200.

[7] , Orientals, arXiv:math.CT/0601383, 2006.

[8] R. Street, The algebra of oriented simplexes, J. Pure Appl. Algebra 49 (1987), no. $3,283-335$.

[9] - The petit topos of globular sets. Category theory and its applications, J. Pure Appl. Algebra 154 (2000), no. 1-3, 299-315.

Richard Steiner r.steiner@maths.gla.ac.uk

Department of Mathematics

University of Glasgow

University Gardens

Glasgow

Scotland G12 8QW

This article is available at http://intlpress .com/HHA/v9/n1/a18 